\title{
Dança sênior: um recurso na intervenção terapêutico-ocupacional junto a idosos hígidos
}

\author{
Janine Gomes Cassiano*; Larissa da Silva Serelli**; Simone Abrantes Cândido*; \\ Aline Torquetti ${ }^{\star \star *}$; Karina Fonseca* ${ }^{\star \star *}$
}

\section{Resumo}

Por meio de relato de caso e levantamento de literatura, visa-se apresentar a dança sênior (DS) como um recurso na intervenção terapêutico-ocupacional para a promoção de saúde em idosos hígidos. A DS é uma atividade grupal que envolve música e atividade física, trabalhando o corpo por meio de coreografias criadas com músicas instrumentais e movimentos ritmados. Objetiva a estimulação cognitiva e sensóriomotora, favorecendo a autoestima e a integração do grupo. A DS foi realizada uma vez por semana junto aos idosos do Projeto "Vale a pena viver", vinculado à Universidade Federal de Minas Gerais. Após um ano de aplicação, solicitou-se a cada participante um depoimento de sua percepção quanto à sua participação na atividade. Realizou-se uma busca no MedLine visando à realização de uma interlocução entre os achados da literatura e a análise dos relatos. Os benefícios da utilização da DS como recurso terapêutico foram observados, concluindo-se que propicia atividade física, lazer, ludicidade, estimulação cognitiva, desenvolvimento da coordenação motora, autoestima e socialização. Os ganhos obtidos com o uso contínuo da dança nas esferas física, cognitiva e social contri- buem para a promoção da percepção da qualidade de vida dos participantes, sugerindo que a DS pode ser utilizada para facilitar a socialização, o conhecimento corporal, estimular a criatividade, a memória e a coordenação motora.

Palavras-chave: Terapia pela dança. Saúde. Idosos.

\section{Introdução}

A longevidade cada vez maior da população justifica as inúmeras pesquisas produzidas hoje na área da gerontologia. A questão é de que forma vivenciar os anos adicionais com bem-estar, saúde e qualidade de vida, características de um envelhecimento ativo. (OMS, 2005).

O envelhecimento é a extensão lógica dos processos fisiológicos de desenvolvimento. (SPIRDUSO, 2005). Esse processo é acompanhado por alterações físicas e cognitivas, que podem levar a prejuízos de ordem funcional, social, comportamental e emocional dos indiví-

* Terapeuta Ocupacional pela Universidade Federal de Minas Gerais. Doutora em Ciências pela Universidade Federal de São Paulo. Professora Adjunta do Departamento de Terapia Ocupacional da Universidade Federal de Minas Gerais. Endereço para correspondência: Rua Guanhaes, 581/101, Bairro Floresta, Belo Horizonte - MG, CEP 31110-160. E-mail: janinecassiano@gmail.com

** Terapeuta Ocupacional e especialista em Gerontologia Social pela Universidade Federal de Minas Gerais.

**** Terapeuta Ocupacional pela Universidade Federal de Minas Gerais.

$\hookrightarrow$ Recebido em junho de 2008 - Avaliado em março de 2009.

$\longrightarrow$ doi:10.5335/rbceh.2009.019 
duos. (MELLO; TUFIK, 2004; ZIMERMAN, 2000).

Para cada indivíduo a velhice é vivenciada singularmente. Características genéticas, culturais e de recursos, hábitos de vida e comportamentos influenciam nos diferentes ritmos de envelhecimento (ZIMERMAN, 2000; SPIRDUSO, 2005). Gáspari e Schwartz (2005) afirmam que o idoso, assim como o adolescente, enfrenta uma crise de identidade durante a qual é afetado em sua autoestima e na aceitação de si mesmo. Isso se reflete na autonomia, na liberdade, convívio social, afetando a frequência e a qualidade dos relacionamentos interpessoais e dos vínculos afetivos. Os autores apontam a importância de ações educativas que possibilitem ao idoso a elaboração, no coletivo, das questões geradoras dessa "crise", dando-lhe oportunidade de incorporar novas atitudes diante do envelhecimento.

A velhice, se comparada às demais etapas da vida, é caracterizada por um maior tempo livre. Isso se deve, em parte, ao advento da aposentadoria e ao desvencilhamento de outras obrigações sociais. Durante essa fase os suportes sociais encontram-se reduzidos, podendo haver ausência de papéis sociais a serem desempenhados e falta de novos planos e objetivos de vida, o que pode contribuir para o isolamento do indivíduo. (FERRARI, 1996). Diante disso, é reconhecida a importância de o idoso se engajar em novas atividades, de reorganizar o tempo que lhe é disponível, ocupando-o significativamente.

Ações de promoção de saúde são caracterizadas por visar ao aumento da saúde e do bem-estar dos indivíduos, contribuindo para uma melhor percepção da qualidade de vida. (CZERESNIA, 1999). Para a Organização Mundial de Saúde (2005), criar medidas que auxiliem os idosos a se manterem saudáveis é uma necessidade, sendo imprescindível desenvolver políticas e programas de envelhecimento ativo.

Tendo em vista as transformações na vida cotidiana do indivíduo idoso e a necessidade de criar estratégias que agreguem qualidade de vida aos anos vividos por ele, a dança sênior pode ser utilizada como uma estratégia de promoção da percepção de bem-estar e saúde de idosos.

O grupo pode promover a socialização, a qualidade de vida e o exercício da cidadania; propicia a criação de vínculos, a ampliação do universo cultural do idoso, o desenvolvimento de novas habilidades e projetos de vida, o conhecimento e a reflexão sobre o envelhecimento. (CASSIANO et al., 2005). Zimerman e Osório (1997), por considerarem a velhice uma questão social, acreditam que grupos podem promover a reconstrução da identidade.

A DS é uma atividade grupal que cria um ambiente de experiências enriquecedoras, no qual habilidades pessoais e interpessoais podem ser exploradas, reforçando-se a autoestima. Os componentes sensório-motores, tais como a mobilidade, a flexibilidade e coordenação, podem ser trabalhados visando à manutenção ou melhora. Os componentes cognitivos, tais como atenção, nível de concentração e retenção, podem ser otimizados. Essas características, associadas, contribuem 
para o desenvolvimento do indivíduo, bem como para a sensação de bem-estar e saúde.

O objetivo deste trabalho é caracterizar a dança sênior como um recurso terapêutico que pode ser utilizado visando à promoção de saúde de idosos hígidos. Para tanto, buscou-se analisar e descrever os benefícios da dança como uma modalidade de exercício físico grupal, com estratégias de otimização cognitiva, de elaboração de laços sociais e vínculos afetivos.

\section{Metodologia}

A DS é uma das atividades desenvolvidas no projeto de extensão "Vale a pena viver", do Departamento de Terapia Ocupacional da Universidade Federal de Minas Gerais, que visa à manutenção da capacidade funcional e à promoção da qualidade de vida de idosos hígidos da comunidade. A DS foi aplicada durante um ano, com frequência semanal e duração de uma hora, em um grupo de vinte idosos com faixa etária entre 65 e 84 anos.

A equipe de trabalho do projeto observou, empiricamente, mudanças nas esferas social, cognitiva, física e emocional dos participantes e optou por colher relatos destes com o objetivo de iniciar a sistematização do estudo da DS como recurso na intervenção terapêutico ocupacional a idosos hígidos.

Inicialmente, foi realizada uma busca na base de dados MedLine (19952005) com as palavras "dança sênior". Foram localizados dois únicos trabalhos: um estudo qualitativo com idosos insti- tucionalizados (ROMERO et al., 2001) e outro sobre o impacto de um programa de DS no nível de massa óssea de idosos. (KUDLACEK; PIETSCHMANN; BERNECKER, 1997). Posteriormente, foi realizada uma análise da atividade "dança sênior", levantando-se as áreas de desempenho, os componentes envolvidos e o contexto no qual a atividade foi desenvolvida. A partir da análise, foi realizada nova busca na base de dados com os descritores "dança", "grupos" e "promoção de saúde". Desenvolveu-se, então, uma interlocução entre os achados da literatura e a análise dos relatos.

\section{A dança e seus benefícios}

Os declínios do processo de envelhecimento acarretam desafios ao controle emocional e social do indivíduo. $\mathrm{O}$ envelhecimento traz a necessidade de as pessoas se adaptarem à "diminuição de força, capacidade física e saúde; às mortes de cônjuges e amigos; à aposentadoria e à redução nos rendimentos; aos novos papéis sociais; e na idade avançada, à realocação das disposições físicas da sua vida". (SPIRDUSO, 2005).

A dança é considerada uma atividade física. (LEAL; HAAS, 2006). Os benefícios desta modalidade de exercício nas esferas social, física e cognitiva dos praticantes são descritos na literatura. A dança possui um componente social, estimulando o desenvolvimento de laços afetivos e contribuindo para a autoestima e percepção da qualidade de vida. (GUIMARÃES; SIMAS; FARIAS, 2003; FILIPETTO; SEVERO, 1999).

Puggard (1994) verificou que a dança é benéfica para o ganho e manutenção 
do condicionamento físico em pessoas idosas. Todaro (2002) descreve que o ato de dançar exercita, dá flexibilidade, modela o corpo e traz benefícios para a postura e coordenação motora.

A prática de atividade física promove melhora na saúde física e mental dos idosos. É capaz de preservar a massa muscular; prevenir sarcopenia, quedas e manter o pico de massa óssea; prover uma melhora global na função cardiovascular, reduzindo o risco de declínio cognitivo. (LAUTERNSCHLAGER et al., 2004). Drela, Kozdron e Scczpiorski (2004) acrescentam que esse tipo de exercício contribui para a saúde ao modular a síntese de determinadas citocinas que são importantes reguladores da resposta imune.

O processo cognitivo é mais rápido e eficiente em indivíduos fisicamente ativos em razão da melhora na circulação cerebral, alteração na síntese e degradação de neurotransmissores. (SPIRDUSO, 2005). A prática de exercícios físicos moderados apresenta um potencial efeito protetor ao reduzir o ritmo de perdas cognitivas no envelhecimento, proporcionando um menor risco de desordens mentais. (MATSUDO; NETO, 2000; MELLO; TUFIK, 2004).

Embora alguns estudos não apresentem resultados significativos da atividade física na dimensão cognitiva, vários autores realçam a importância do exercício físico na manutenção ou melhora das funções cognitivas e no bem-estar (MELLO; TUFIK, 2004; SPIRDUSO, 2000; SEVERO; SANTOS; DIAS, 2000).

A manutenção do desempenho da memória e de outras funções cognitivas é vital para o envelhecimento bem-sucedido. O treinamento da memória pode melhorar a capacidade de abstração e tempo de reação, as habilidades para resolver problemas e a memorização. (GUARIDO et al., 2003). Segundo Guimarães (2003), atenção, memória, raciocínio e imaginação são funções mentais exercidas e desenvolvidas durante a dança e essas habilidades são incorporadas, com vantagens, no dia a dia.

\section{A dança sênior}

A DS surgiu em 1974, na Alemanha, sob a liderança de Ilse Tutt, onde em 1977 foi fundada a Federação Nacional de Dança Sênior na Alemanha. Foi introduzida no Brasil por Christel Weber em 1978. A partir de 1982 passou a ser aplicada no Ancianato Bethesda, culminando com a criação da Associação de Dança Sênior em 1993, em Pirabeiraba - SC.

Segundo a Associação de Dança Sênior, a DS objetiva a busca da autoestima e a integração do grupo, promovendo o bem-estar dos participantes. A dança trabalha com o corpo através de coreografias criadas a partir de músicas instrumentais e movimentos ritmados. É realizada em grupo e com seus participantes em círculo, ora sentados, ora em pé; ora em pares, em fileiras ou dispersos pelo salão. As coreografias da DS são ensinadas, de forma padronizada, às pessoas que serão dirigentes de novos grupos. Além disso, existem cursos de "reciclagem", nos quais os dirigentes podem aperfeiçoar e aprender novas coreografias. 
ADS é uma atividade voltada para a população idosa que envolve a música e a atividade física; exige dos participantes movimentos amplos, rápidos e lentos, coordenados, simultâneos, ritmados, acompanhando a marcação do dirigente e do grupo. O repertório é diversificado, do ponto de vista de ritmos, coreografias e complexidade de movimentos. Esse fato favorece a adequação da DS ao grupo, às suas demandas físicas, cognitivas $\mathrm{e}$ emocionais, visto que cada idoso realiza os exercícios dentro dos seus limites e capacidade.

Utilizada como recurso terapêutico, a DS possibilita a expressão emocional, favorecendo o pensar criativo e o autoconhecimento. Isso leva o idoso a tomar consciência de sua potencialidade e a (re)aprender uma nova forma de viver. Segundo Peto (2000), ainda há falta de conhecimento sistematizado a respeito da dança como instrumento de valor terapêutico e educativo.

A DS pode proporcionar benefícios físicos, cognitivos, sociais e emocionais. Dentre os físicos estão a mobilização, a flexibilidade e a coordenação motora; nos cognitivos, a atenção e a memória são componentes trabalhados com a prática contínua da dança; dentre os sociais, o grupo favorece a interação, a expressão e o sentimento de pertencimento dos participantes. A DS ainda pode ser considerada uma modalidade de lazer/recreação realizada em grupo, sendo mediadora de relações humanas.

Kudlacek, Pietschmann e Bernecker (1997) investigaram o impacto dessa dança na manutenção da massa óssea em mulheres maduras e idosas. O estudo foi prospectivo, com 28 senhoras de idades entre 46 e 78 anos. Divididas em dois grupos, conforme a presença ou ausência de osteoporose, as participantes foram submetidas a um programa regular de DS durante 12 meses. Os autores constataram que os movimentos da dança para idosas sem osteoporose exercem um efeito benéfico sobre o controle do peso, flexibilidade das articulações e manutenção da massa óssea. As idosas que apresentavam osteoporose obtiveram um significativo aumento da massa óssea durante a prática regular da DS. Os resultados comprovam o benefício da prática regular dessa modalidade de exercício na manutenção e/ou aumento da massa óssea em mulheres maduras e idosas.

Um outro estudo, de natureza qualitativa, objetivando analisar o impacto da DS em idosos institucionalizados, foi realizado por Romero et al. (2001). Uma amostra de vinte idosos de ambos os sexos e idades entre sessenta e noventa anos foi submetida à DS duas vezes por semana durante dez meses. Os autores constataram que a dança proporcionou uma mudança no comportamento dos idosos, estimulando a solidariedade, promovendo a motivação, ajudando na interação entre os participantes, proporcionando-lhes, enfim, uma maior qualidade de vida.

ADS estimula a atenção, já que para aprender novos passos da dança o participante tem de se manter em "estado de alerta", pois a "atividade requer certo grau de atenção sustentada durante o período que se leva para completá-la." (GRIEVE, 2005). Trabalha, ainda, a me- 
mória, ao exigir do idoso a capacidade de reter e resgatar as coreografias.

O esquema corporal e a coordenação motora também podem ser trabalhados nessa dança, uma vez que estimula a representação mental das partes do corpo e de sua relativa posição dentro do espaço ao promover movimentos controlados, segundo um ritmo previamente estabelecido, de modo associado e dissociado, dos membros superiores e/ou inferiores para realizar parte de uma coreografia.

Outro elemento da cognição trabalhado na DS é o planejamento mental envolvido na execução das coreografias. Há uma exigência de atenção do participante, que vai desde o processamento automático das coreografias realizadas até o processamento controlado, necessário para se aprender uma nova coreografia.

A DS, por ser uma atividade grupal, possibilita o desenvolvimento de relações interpessoais, promovendo a socialização. Para Severo (2000), os movimentos corporais da dança contribuem para a interação entre um participante e o grupo, pois favorecem o contato e a interação social. Zimerman e Osório (1997) afirmam que todo ser humano necessita de formas de comunicação, integração e reconhecimento de seus pares, por se tratar de um ser gregário. As atividades desenvolvidas em grupo, segundo Ferrigno, Leite e Abigalil (2006), exercem no idoso um poder restaurador da afetividade, da autoestima, da autoconfiança e até um sentimento de capacidade de retorno à atividade laborativa.

\section{Projeto "Vale a pena viver"}

Manter e melhorar a percepção da qualidade de vida é um dos objetivos da terapia ocupacional junto à população idosa. A DS é um recurso terapêutico rico para esse fim, pois a ludicidade, permeando o desenvolvimento da coordenação motora e a interação grupal, facilita o sentimento de pertencimento. Esses aspectos puderam ser observados durante os encontros com os participantes do projeto "Vale a pena viver", como pode ser percebido nos seguintes relatos:

A dança traz alegria a pessoas que foram frustradas a vida toda, cuidou da família, trabalhou. Ela traz cidadania, recupera os valores da gente. Ajuda a comunicação. Recupera a comunicação perdida, o entrosamento, e com isso melhora a saúde, menos depressão, mais necessidade de ajudar o próximo. (L. E. N., 71 anos)

A dança trouxe facilidade de movimentar mais, tenho mais agilidade e aqui me sinto muito feliz. (A. S. S., 74 anos)

A dança desenvolve tanto atividade física, quanto mental. (N. B. S., 76 anos)

Muito bom para a minha vida: me sinto mais feliz, mais tranquila. É um vínculo de amizade que a gente tem! (A. A. R., 77 anos)

É uma atividade que atrai, traz benefícios físicos e ajuda na memória. ( M. N., 79 anos).

Depois que entrei no projeto minha vida mudou muito. Agora, saio mais vezes de casa e nos dias que tenho o projeto fico animada, doida que chegue a hora de ir para as atividades. Sinto que minha cabeça está melhor, estou pegando o ônibus sozinha. Nas horas que estou com as colegas de projeto fico muito feliz, escuto muitos casos e rimos das coisas que acontecem. Com o projeto estou 
conversando um pouco mais com as pessoas, conto das atividades que aprendi e fiz. Gosto das atividades para a memória e da dança; a ginástica tem feito muito bem para mim e aprendo muitos movimentos diferentes. (A. A. O., 84 anos)

Silêncio! Atenção! Olhem todos para cá. Sorriam! A dança sênior vai começar! Coluna ereta. Elegância a primar. Mão direita com direita, o vizinho cumprimentar! Agora, do outro lado, um beijo vamos jogar! Mãos para cima. Pés para o ar. De novo, quieto em seu lugar. Chegou a hora de se levantar! E de mãos dadas bailar... bailar... Um, dois e tip! Três, quatro e vai! Dance para lá, dance pra cá! Até a música acabar! Nossa alma de alegria. Repleta já está. Os problemas... Que problemas? As dores... Viraram flores. Vamos dançar e aplaudir. E com o coração cheio de amores, viver a cantar, a agradecer e a sorrir! (Poesia de uma das participantes sobre a dança sênior - M. P. E, 65 anos)

\section{Considerações finais}

A DS é um agente facilitador de maior adesão dos indivíduos idosos à prática de uma atividade física, podendo produzir tanto benefícios físicos e cognitivos quanto emocionais e sociais.

O uso da DS como recurso no atendimento terapêutico ocupacional a idosos hígidos propicia autonomia, autoconhecimento e ressignificação do cotidiano. Dessa maneira, é possível concluir que o grupo de DS realizado no projeto "Vale a pena viver" pode ser considerado um dispositivo terapêutico, na medida em que facilita e favorece a vivência grupal, ampliando o suporte social e estimulando as funções físicas e cognitivas, gerando também benefícios emocionais e sociais.
Senior dance: a resource in occupational therapy intervention with healthy elderly

\section{Abstract}

By means of a case report and survey of the literature, this study intended to introduce the Senior dance (SD) as a resource in occupational therapy interventions for health promotion with the elderly. SD is a group activity, that involves music and physical activity, working the body through creative choreographies with instrumental music and rhythmic movements. Its objective is cognitive, sensory and motor stimulation, favoring self-esteem and group integration. The SD sessions were carried out once a week with elderly participants in a community project of the Universidade Federal de Minas Gerais, entitled: "It is worth living". After one year, the participants were requested to provide information about their perceptions of participation in this activity. A Medline search was also performed with the objective of linking the findings of the literature with the analysis of these cases. The benefits of employing the SD were observed as a therapeutic resource to promote physical activity, leisure, and cognitive stimulation, as well as the development of motor coordination, self-esteem and socialization. The gains obtained with the continuous employment of the SD in the physical, cognitive, and social spheres may have contributed to the promotion of the perceptions of the quality of life of the participants, suggesting that SD could be used to facilitate socialization, body image and stimulate creativity, memory and motor coordination.

Key words: Senior dance. Health. Elderly. 


\section{Referências}

ASSOCIAÇÃO DE DANÇA SÊNIOR. $O$ que é dança sênior? Disponível em: <www.portalbethesda.org.br/danca_senior.htm>. Acesso em: 11 jul. 2007.

CASSIANO, J. C. et al. Promovendo saúde e qualidade de vida em adultos maduros e idosos. In: ENCONTRO DE EXTENSÃO DA UFMG, 8, Belo Horizonte, 2005. Anais eletrônicos... Belo Horizonte: UFMG, 2005. Disponível em: <www.ufmg.br/proex/ arquivos/8Encontro/Indice2.htm>. Acesso em: 17 jul. 2007.

CZERESNIA, D. O conceito de saúde e a diferença entre prevenção e promoção. Cadernos Saúde Pública, São Paulo, v. 15, n. 4, p. 701-709, 1999.

DRELA, N. D.; KOZDRON, E.; SCCZPIORSKI, P. Moderate exercise may attenuate some aspects of immunosenescence. BMC Geriatrics, v. 4, n. 8, p. 1-7, 2004. Disponível em: <www.biomedcentral.com/1471-2318/4/8>. Acesso em: 11 jul. 2007.

FERRARI, M. A. C. Lazer e ocupação do tempo livre. In: PAPALEO NETTO, M. (Org.). Gerontologia. São Paulo: Atheneu, 1996. p. 98-106.

FERRIGNO, J. C.; LEITE, M. L. C. B.; ABIGALIL, A. P. C. Centros e grupos de convivência de idosos: da conquista do direito ao lazer ao exercício da cidadania. In: FREITAS, E. V. et al. (Org.). Tratado de geriatria e gerontologia. 2. ed. Rio de Janeiro: Guanabara Koogan, 2006. p. 1436-1443.

FILIPETTO, M. C.; SEVERRO, C. Novas perspectivas: dança e expressão corporal para idosos. Cadernos Adulto, Santa Maria, n. 3, p. 137-138, 1999.
GÁSPARI, J. C.; SCHWARTZ, G. M. O idoso e a ressignificação emocional do lazer. Psicologia - Teoria e Pesquisa, Brasília, v. 21, n. 1, p. 69-76, 2005.

GRIEVE, J. Neuropsicologia em terapia ocupacional: exame da percepção e cognição. 2. ed. São Paulo: Santos, 2005.

GUARIDO, E. L. et al. Desempenho cognitivo no idoso: desenvolvimento de uma proposta de intervenção. Ciência Médica, Campinas, v. 12, n. 4, p. 287-297, 2003.

GUIMARÃES, A. C. A.; SIMAS, J. P. N.; FARIAS, S. F. Dança como uma contribuição para a qualidade de vida. Cinergis, Santa Cruz do Sul, v. 4, n. 1, p. 29-37, 2003.

KUDLACEK, S.; PIETSCHMANN, F.; BERNECKER, P. et al. The impact of a senior dancing program on spinal and peripheral bone massi. Phys. Med. Rehabil., v. 76, n. 6, p. $477-481,1997$.

LAUTENSCHLAGER, N. T. et al. Can physical activity improve the mental health of older adults? Annals of General Hospital Psychiatry, v. 3, n. 12, p. 1-5, 2004.

LEAL, I. J.; HAAS, A. N. O significado da dança na terceira idade. Revista Brasileira de Ciências do Envelhecimento Humano, Passo Fundo, v. 3, n. 1, p. 64-71, jan./jun. 2006.

MATSUDO, S. M.; NETO, T. L. B. Efeitos benéficos da atividade física na aptidão física e saúde mental durante o processo de envelhecimento. Revista Brasileira de Atividade Física em Saúde, Florianópolis, v. 5, n. 2, p. 60-76, 2000.

MELLO, M. T.; TUFIK, S. Atividade física, exercício físico e aspectos psicobiológicos. Rio de Janeiro: Guanabara Koogan, 2004.

ORGANIZAÇÃO MUNDIAL DE SAUDE. Envelhecimento ativo: uma política de saúde. Brasília: OPAS, 2005. 
PETO, A. C. Terapia através da dança com laringectomizados. Relato de experiência. Revista Latino America de Enfermagem, Ribeirão Preto, v. 23, n. 6, p. 110-113, 2000.

PUGGARD, L. Physical conditioning in elderly people. Scand. J. Med. Sci. Sports, v. 4, p. 47-56, 1994.

ROMERO, E. et al. Senior dance pedagogy with elderly in a nursing home as a factor of socialization, motivation and quality of life. WORD CONGRESS OF SPORT PSYCHOLOGY, 10 th. 2001, Skiathos. Proceedings... Thessaloniki: Christodoulidi Publications, 2001. p. 160-16.

SEVERO, C.; SANTOS, D. L.; DIAS, J. F. S. A influência da dança e da musicalidade nos estados de ânimo dos idosos. Cadernos Adulto, Santa Maria, n. 4, p. 35-42, 2000.

SPIRDUSO, W. W. Dimensões físicas do envelhecimento. Barueri: Manole, 2005.

TODARO, M. A. Dança e envelhecimento: conceitos, pesquisas e experiências. A Terceira Idade, São Paulo, v. 13, n. 24, p. 28-37, 2002.

ZIMERMAN, D. E.; OSÓRIO, L. C. Como trabalhamos com grupos. Porto Alegre: ArtMed, 1997.

ZIMERMAN, G. I. Aspectos biopsicossociais. Rio Grande do Sul: Artmed, 2000. 